Alexander Mikhaylov (St. Petersburg)

JoAnNa RENCEAWOWICZ (Warszawa)

WOJCIECH M. ZAJĄCZKOWSKI (Warszawa)

\title{
ON GLOBAL REGULAR SOLUTIONS TO THE MHD EQUATIONS IN A SMOOTH TOROIDAL DOMAIN
}

Abstract. We consider the magnetohydrodynamic equations in a smooth toroid located at a positive distance from its axis of symmetry. Since the domain is axially symmetric, we can prove existence of global regular axially symmetric solutions. Next, stability of these solutions is proved. In this way the existence of global regular solutions close to the axially symmetric solutions for all time is shown.

1. Introduction. We examine the magnetohydrodynamic (mhd) motions (see [C, LLP]) in an axially symmetric domain $\Omega \subset \mathbb{R}^{3}$ of smooth toroidal shape, located at a positive distance from its axis of symmetry. The mhd equations are

$$
\begin{array}{ll}
v_{t}+v \cdot \nabla v-\nu \Delta v+\nabla q-H \cdot \nabla H=f & \text { in } \Omega^{T} \equiv \Omega \times(0, T), \\
\operatorname{div} v=0 & \text { in } \Omega^{T}, \\
H_{t}+v \cdot \nabla H-H \cdot \nabla v-\mu \Delta H=0 & \text { in } \Omega^{T}, \\
\operatorname{div} H=0 & \text { in } \Omega^{T},
\end{array}
$$

where $v=\left(v_{1}(x, t), v_{2}(x, t), v_{3}(x, t)\right) \in \mathbb{R}^{3}$ is the velocity of the fluid, $q=$ $p(x, t)+\frac{1}{2} H^{2}(x, t) \in \mathbb{R}$ is the total pressure, $H=\left(H_{1}(x, t), H_{2}(x, t), H_{3}(x, t)\right)$ $\in \mathbb{R}^{3}$ is the magnetic field, $f=\left(f_{1}(x, t), f_{2}(x, t), f_{3}(x, t)\right) \in \mathbb{R}^{3}$ is the external force, $x=\left(x_{1}, x_{2}, x_{3}\right)$ is the Cartesian system of coordinates, $\nu>0$ is the constant viscosity coefficient and $\mu>0$ is the resistivity.

2010 Mathematics Subject Classification: 35B07, 35B35, 35B40, 76W05.

Key words and phrases: magnetohydrodynamics, stability of axially symmetric solutions, global existence of regular solutions.

Received 4 November 2015; revised 10 January 2017.

Published online 8 June 2017. 
Let $S=\partial \Omega$ be the boundary of $\Omega$. We add the following boundary conditions:

$$
\begin{aligned}
& \left.v\right|_{S}=0, \\
& \left.H \cdot \bar{n}\right|_{S}=0, \quad \bar{n} \times\left.\operatorname{rot} H\right|_{S}=0,
\end{aligned}
$$

where $\bar{n}$ is the unit outward normal vector to $S$. Moreover, we need the initial conditions

$$
\left.v\right|_{t=0}=v(0),\left.\quad H\right|_{t=0}=H(0) \quad \text { in } \Omega .
$$

To describe $\Omega$, which is an axially symmetric domain, we need the cylindrical coordinates $(r, \varphi, z)$ introduced by the relations

$$
x_{1}=r \cos \varphi, \quad x_{2}=r \sin \varphi, \quad x_{3}=z .
$$

Then the cylindrical coordinates of the vectors $v$ and $H$ are defined with the help of the vectors

$$
\bar{e}_{r}=(\cos \varphi, \sin \varphi, 0), \quad \bar{e}_{\varphi}=(-\sin \varphi, \cos \varphi, 0), \quad \bar{e}_{z}=(0,0,1)
$$

in the following way:

$$
u_{r}=u \cdot \bar{e}_{r}, \quad u_{\varphi}=u \cdot \bar{e}_{\varphi}, \quad u_{z}=u \cdot \bar{e}_{z},
$$

where $u$ replaces any vector in this paper, and the dot denotes the scalar product in $\mathbb{R}^{3}$.

To characterize $\Omega$ we introduce a plane $P(\varphi)$ which contains the $x_{3}$-axis and makes angle $\varphi$ to the plane $x_{2}=0$. Let $\psi(r, z)=0$ be a closed smooth curve in the plane $P(\varphi)$ such that $r \in(a, b)$ where $0<a<b$. Then our domain is such that

$$
\begin{aligned}
& P(\varphi) \cap \Omega=\{(r, z): \psi(r, z)<0\}, \quad \varphi \in[0,2 \pi], \\
& \min \left\{\operatorname{dist}\left(P(\varphi) \cap \Omega, x_{3} \text {-axis }\right)\right\}>0 .
\end{aligned}
$$

This means that $\Omega$ is a rotation of the set $\{(r, z): \psi(r, z)<0\}$ around the $x_{3}$-axis.

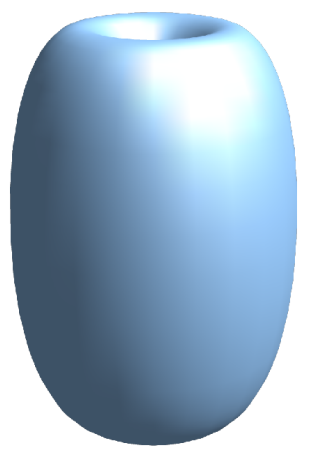

Fig. 1. Domain $\Omega$ : toroid with an ellipse cross section 
Additionally, for fixed $\varphi$, we set $\Omega_{0}=P(\varphi) \cap \Omega, S_{0}=\partial \Omega_{0}$. Then

$$
\left.\bar{n}\right|_{S_{0}}=\frac{\nabla \psi}{|\nabla \psi|}=\frac{1}{|\nabla \psi|}\left[\frac{\psi_{, r}}{r}\left(\begin{array}{c}
x_{1} \\
x_{2} \\
0
\end{array}\right)+\psi_{, z}\left(\begin{array}{l}
0 \\
0 \\
1
\end{array}\right)\right],
$$

where $|\nabla \psi|=\sqrt{\psi_{, r}^{2}+\psi_{, z}^{2}}$.

As an example of a curve as above we have

$$
\psi(r, z)=\left(r-a_{0}\right)^{2}+z^{2}-r_{0}^{2}=0,
$$

where $a_{0}>r_{0}$, so $r \in\left[a_{0}-r_{0}, a_{0}+r_{0}\right]$ and $|z| \leq r_{0}$, and then the domain $\Omega$ is a torus.

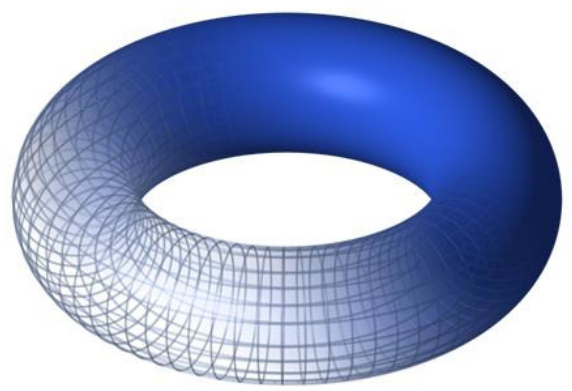

Fig. 2. Domain $\Omega$ : torus

The boundary condition (1.2) is the non-slip condition. Assuming that the magnetic induction $B$ is equal to $\mu_{0} H$, where $\mu_{0}$ is the magnetic vacuum permeability, the first boundary condition of (1.3) means that the flux of the magnetic induction through the boundary $S$ does not appear. Since $j=\operatorname{rot} H$ is a current, the second boundary condition of (1.3) means that the tangent coordinates of $j$ to $S$ vanish. Hence, there is no restriction on the normal coordinate of $j$. It seems that this stabilizes the motion inside $\Omega$. Physically, the problem is a very first step to the famous tokamak fusion, where the tokamak is a device that uses a powerful magnetic field to confine plasma to a torus shape region. Moreover, the protons in the CERN cyclotron located near Geneva move in a domain similar to the one considered in this paper, where, however, the motion is controlled by an external magnetic field. Therefore, the current flux through $S$ must disappear. In that case we have a free boundary problem which is much more complicated than the problem examined in this paper and requires a different approach.

Our aim is to prove existence of global regular solutions to problem (1.1)-(1.4) without assumptions on smallness of the initial data and the external force. For this purpose we first consider problem (1.1)-(1.4) in the 
domain $\Omega_{0}$, where $v=v_{s}=\left(v_{r}(r, z, t) \bar{e}_{r}+v_{\varphi}(r, z, t) \bar{e}_{\varphi}+v_{z}(r, z, t) \bar{e}_{z}\right), H=$ $H_{s}=\left(H_{r}(r, z, t) \bar{e}_{r}+H_{\varphi}(r, z, t) \bar{e}_{\varphi}+H_{z}(r, z, t) \bar{e}_{z}\right), f=f_{s}=\left(f_{r}(r, z, t) \bar{e}_{r}+\right.$ $\left.f_{\varphi}(r, z, t) \bar{e}_{\varphi}+f_{z}(r, z, t) \bar{e}_{z}\right), q=q_{s}(r, z, t)=p_{s}(r, z, t)+\frac{1}{2} H_{s}^{2}(r, z, t)$ satisfy

$$
v_{s t}+v_{s} \cdot \nabla v_{s}-\nu \Delta v_{s}+\nabla q_{s}-H_{s} \cdot \nabla H_{s}=f_{s},
$$

$\operatorname{div} v_{s}=0$,

$H_{s t}+v_{s} \cdot \nabla H_{s}-H_{s} \cdot \nabla v_{s}-\mu \Delta H_{s}=0$,

$\operatorname{div} H_{s}=0$

and

$$
\begin{aligned}
& \left.v_{s}\right|_{S_{0}}=0,\left.\quad \bar{n} \cdot H_{s}\right|_{S_{0}}=0, \quad \bar{n} \times\left.\operatorname{rot} H_{s}\right|_{S_{0}}=0, \\
& \left.v_{s}\right|_{t=0}=v_{s}(0),\left.\quad H_{s}\right|_{t=0}=H_{s}(0) .
\end{aligned}
$$

In Section 3 we prove existence of global regular solutions to problem (1.8)(1.10).

To show stability of these axi-symmetric solutions we introduce the quantities

$$
u=v-v_{s}, \quad K=H-H_{s}, \quad g=f-f_{s}, \quad d=q-q_{s}
$$

which are solutions to the problems

$$
\begin{aligned}
\begin{aligned}
u_{t}-\nu \Delta u+\nabla d= & -u \cdot \nabla u-u \cdot \nabla v_{s}-v_{s} \cdot \nabla u+K \cdot \nabla K \\
& +K \cdot \nabla H_{s}+H_{s} \cdot \nabla K+g,
\end{aligned} & \begin{aligned}
\operatorname{div} u=0
\end{aligned}
\end{aligned}
$$

and

$$
\begin{aligned}
K_{t}-\mu \Delta K= & -u \cdot \nabla K-u \cdot \nabla H_{s}-v_{s} \cdot \nabla K+K \cdot \nabla u \\
& +K \cdot \nabla v_{s}+H_{s} \cdot \nabla u, \\
\operatorname{div} K=0 . &
\end{aligned}
$$

Moreover, we add the boundary conditions

$$
\left.u\right|_{S_{0}}=0,\left.\quad K \cdot \bar{n}\right|_{S_{0}}=0, \quad \bar{n} \times\left.\operatorname{rot} K\right|_{S_{0}}=0
$$

and initial conditions

$$
\left.u\right|_{t=0}=u(0),\left.\quad K\right|_{t=0}=K(0) .
$$

There is a vast literature concerning regularity of solutions to incompressible magnetohydrodynamics (mhd). We can distinguish the following directions:

1. Three-dimensional mhd (either with small data or close to some special solutions) [XZ, HX1, [HX2, LZ, CMZ, [NZ, NSZ].

2. Two-dimensional mhd [LXZ, DL, Z, , CW, ST,

3. Mhd without resistivity [XZ, LXZ, L]. 
4. Axially symmetric mhd [L,, $\mathrm{NZ}]$. Recall that the existence of axi-symmetric nonswirl solutions to the Navier-Stokes equations in a cylindrical domain was first proved by Ladyzhenskaya [La1].

Our aim in this paper is to prove global existence of regular solutions to the mhd problem (1.1)-(1.4) in a three-dimensional domain. Since the regularity problem and regularity of axi-symmetric solutions with large swirl to the Navier-Stokes equations are still open problems, we consider problem (1.1)-(1.4) in a torus around the axis of symmetry. Our domain is located at a positive distance from the axis of symmetry, i.e. the $x_{3}$-axis. Therefore, in such domains, we can prove existence of global regular axisymmetric solutions to the mhd problem (1.8)-(1.10) with large swirl $r v_{\phi}$. The existence of these solutions is proved in Section 3. In Lemmas 3.1 and 3.2, applying the step by step in time arguments from [ZZ1, ZZ2, Z1, Z2] we find a global estimate for $\left\|v_{s}, H_{s}\right\|_{V_{2}^{1}(\Omega \times(k T, t))}, t \in(k T,(k+1) T], k \in \mathbb{N}_{0}$, where $T>0$ is fixed (see Theorem 1). The above estimate is so strong that application of the technique of Sobolev spaces with a mixed norm (see Lemma 2.4) shows that $v_{s}, H_{s}$ belong to $W_{\sigma, 2}^{2,1}, \sigma>1$ (see Theorem 2).

Next, we look for solutions close in appropriate norms to the axi-symmetric solutions (see problem (1.11)-(1.15)). To show existence of global regular solutions to this problem we need smallness of initial data (see (1.15)) and of the external force $g$ (see (1.11)). The existence of these solutions is proved in Section 4. This is treated as stability of the axi-symmetric solutions. The results are formulated in Theorems 3 and 4. Assuming that the external forces do not vanish in time, we are looking for solutions non-decaying in time. This is the reason why the step by step in time technique is used (see [ZZ1, ZZ2, Z1, Z2]). This approach enables one to prove existence of stationary solutions via stability. In this paper the $L_{2}$-technique is used because almost all estimates are obtained by the energy method. Besides the obvious physical background, the axi-symmetric solutions in a torus are in fact two-dimensional because the axis of symmetry is outside the torus. The existence of regular axi-symmetric solutions in that type of domains is shown in [Z3], and global regular existence of two-dimensional solutions was proved by Ladyzhenskaya in her famous paper [La2].

In Section 2 we introduce notation and recall some auxiliary results. In Section 3 we prove global existence of regular axi-symmetric solutions to (1.8)-(1.10). In Section 4 the existence of global regular solutions to problem (1.12)-(1.15) is proved for sufficiently small $\|u(0), K(0)\|_{H^{1}}$ and $\|g(t)\|_{L_{2}}$, $t \in \mathbb{R}_{+}$. Then stability follows from the following implication:

$$
\|u(0), K(0)\|_{H^{1}} \leq \gamma \quad \text { implies } \quad\|u(t), K(t)\|_{H^{1}} \leq \gamma
$$

with $\gamma$ sufficiently small and $t \in \mathbb{R}_{+}$. 
In this way we have the existence of global regular solutions to problem (1.1)-(1.4) which are close to the axi-symmetric solutions determined by problem (1.8)-(1.10).

Now we present the main results of this paper. From Lemmas 3.1 and 3.2 (see proofs) we have

TheOREM 1. Assume $v_{s}(0), H_{s}(0) \in H^{1}\left(\Omega_{0}\right)$ and $f_{s} \in L_{2}(k T,(k+1) T$; $\left.L_{2}\left(\Omega_{0}\right)\right)$ for all $k \in \mathbb{N}_{0}$. Assume that $T$ is such that

$$
\begin{aligned}
\nu_{*} c_{s 2} T / 2 \geq\left[\left(1+\frac{1}{c_{s 1}\left(1-\exp \left(-\nu_{*} c_{s 1} T\right)\right)}\right) \frac{1}{\nu} \sup _{k \in \mathbb{N}_{0}}\right. & \int_{k T}^{(k+1) T}\left\|f_{s}(t)\right\|_{L_{2}\left(\Omega_{0}\right)}^{2} d t \\
& \left.+\left\|v_{s}(0), H_{s}(0)\right\|_{L_{2}\left(\Omega_{0}\right)}^{2}\right]^{2},
\end{aligned}
$$

where $\nu_{*}=\min \{\nu, \mu\}$, the constant $c_{s 1}$ appears in (3.4) and $c_{s 2}$ in (3.8). Then there exists a solution $\left(v_{s}, H_{s}\right)$ to problem (1.8)-(1.10) such that $\left(v_{s}, H_{s}\right) \in$ $V_{2}^{1}\left(\Omega_{0} \times(k T,(k+1) T)\right)$ for all $k \in \mathbb{N}_{0}$ and $\left\|v_{s}, H_{s}\right\|_{V_{2}^{1}\left(\Omega_{0} \times(k T,(k+1) T)\right)}$ is bounded by a constant depending on the data but not on $k$.

Proof. The assumptions of this theorem follow from the assumptions of Lemmas 3.1 and 3.2. Estimates (3.2) and (3.6) imply the assertion of Theorem 1 and conclude the proof.

Theorem 1 and Lemma 3.3 yield

TheOREM 2. Let the assumptions of Theorem 1 hold. Let $\left(v_{s}(0), H_{s}(0)\right) \in$ $B_{2, \sigma}^{2-2 / \sigma}\left(\Omega_{0}\right)$ and $f_{s} \in L_{2}\left(k T,(k+1) T ; L_{\sigma}\left(\Omega_{0}\right)\right)$ with $\sigma \in(2, \infty)$ for all $k \in \mathbb{N}_{0}$. Then there exists a solution $\left(v_{s}, H_{s}\right)$ to problem $(1.8)-(1.10)$ in $W_{\sigma, 2}^{2,1}\left(\Omega_{0} \times(k T,(k+1) T)\right)$ for all $k \in \mathbb{N}_{0}$, and

$$
\left\|v_{s}, H_{s}\right\|_{W_{\sigma, 2}^{2,1}\left(\Omega_{0} \times(k T,(k+1) T)\right)}, \quad k \in \mathbb{N}_{0},
$$

is bounded by a constant independent of $k$.

Proof. The aim of this theorem is to increase regularity of solutions obtained in Theorem 1. The technique is established in Lemma 3.3. In view of (3.6) the increase of regularity in the interval $[0, T]$ is shown in (3.11), where the theory of Sobolev spaces with mixed norm is used. To extend the estimate to all time intervals $[k T,(k+1) T], k \in \mathbb{N}_{0}$, we introduce the cut-off function $\zeta_{k}=\zeta_{k}(t)$ defined below (3.11). Next, we derive a localized in time problem (3.12). Consequently, by the step by step in time argument we show (3.13) and (3.14). Thus, the statement of the theorem holds.

To formulate results on stability we examine problem (1.12)-(1.15). 
TheOrem 3. Let the assumptions of Theorem 2 be satisfied. Assume that $\gamma_{*}$ is a number such that $\gamma_{*} \leq \sqrt[4]{\nu_{*}^{2} / 2}, \nu_{*}=\min \{\nu, \mu\}$. Assume that

$$
\begin{aligned}
& \|\nabla u(0), \operatorname{rot} K(0)\|_{L_{2}(\Omega)}^{2} \leq \gamma, \\
& \frac{c_{3}}{\nu_{*}}\left\|v_{s}(t), H_{s}(t)\right\|_{W_{3}^{1}\left(\Omega_{0}\right)}^{4} B_{3}^{2}+\|g(t)\|_{L_{2}(\Omega)}^{2} \leq \nu_{*} c_{2} \frac{\gamma}{4},
\end{aligned}
$$

where $\gamma \leq \gamma_{*}$, the constants $c_{2}, c_{3}$ are introduced in (4.19), (4.20), respectively, $B_{3}$ is defined in (4.2), and $\left\|v_{s}(t), H_{s}(t)\right\|_{W_{3}^{1}\left(\Omega_{0}\right)}$ is estimated in Theorem 2. Then

$$
\|\nabla u(t), \operatorname{rot} K(t)\|_{L_{2}(\Omega)}^{2} \leq \gamma \quad \text { for any } t \in \mathbb{R}_{+} .
$$

Proof. In Lemma 4.1 we prove in (4.2) that

$$
\|u(t), K(t)\|_{L_{2}} \leq c\left(A_{6}\right)\left(B_{1}+B_{2}\right),
$$

where $A_{6}$ is introduced in (3.6) and $B_{1}, B_{2}$ in (4.1); we also estimate the $H^{1}$ norm of $u(t), K(t)$ by $B_{3}$. The estimate holds for all $t \in \mathbb{R}_{+}$. Assuming that $\gamma$ is so small that $\gamma \leq \gamma_{*} \leq \sqrt[4]{\nu_{*}^{2} / 2}$ we show the assertion of Theorem 3 by a contradiction argument in Lemma 4.2. This ends the proof.

TheOREM 4. Let the assumptions of Theorems 2 and 3 be satisfied. Then there exists a global regular solution to problem (1.1)-(1.4) in the form

$$
v=v_{s}+u, \quad H=H_{s}+K,
$$

where $\left(v_{s}, H_{s}\right)$ is described by Theorem 2 and $(u, K)$ by Theorem 3 .

Proof. In view of problems (1.8)-(1.10) and (1.12)-(1.15) the functions defined in (1.16) are solutions to problem (1.1)-(1.4). Existence of solutions to problem (1.8)-(1.10) follows from the Faedo-Galerkin method and estimates proved in Lemmas 3.1 and 3.2. By Lemma 3.3 the solutions $v_{s}$ and $H_{s}$ are regularized via the theory of Sobolev spaces with mixed norm up to $W_{\sigma, 2}^{2,1}, \sigma>3$. The existence of solutions to (1.12)-(1.15) follows by applying a fixed point argument (for example, the Leray-Schauder theorem) for $\gamma$ sufficiently small. This ends the proof.

2. Notation and auxiliary results. By $L_{p}(\Omega), p \in[1, \infty]$, we denote the Lebesgue space of $p$-integrable functions. By $H^{s}(\Omega), s \in \mathbb{N}_{0}=\mathbb{N} \cup\{0\}$, we denote the Sobolev space of functions with the finite norm

$$
\|u\|_{H^{s}} \equiv\|u\|_{H^{s}(\Omega)}=\sum_{|\alpha| \leq s}\left(\int_{\Omega}\left|D_{x}^{\alpha} u\right|^{2} d x\right)^{1 / 2},
$$

where $D_{x}^{\alpha}=\partial_{x_{1}}^{\alpha_{1}} \partial_{x_{2}}^{\alpha_{2}} \partial_{x_{3}}^{\alpha_{3}},|\alpha|=\alpha_{1}+\alpha_{2}+\alpha_{3}, \alpha_{i} \in \mathbb{N}_{0}, i=1,2,3$.

It is convenient in mhd to use the notation

$$
\|u, v\|_{X}^{2}=\|u\|_{X}^{2}+\|v\|_{X}^{2}
$$

where $X$ is any function space used. 
By $V_{2}^{k}\left(\Omega \times\left(T_{1}, T_{2}\right)\right)$ we denote the space of functions with

$$
\|u\|_{V_{2}^{k}\left(\Omega \times\left(T_{1}, T_{2}\right)\right)}=\left(\operatorname{ess~sup}_{t \in\left(T_{1}, T_{2}\right)}\|u(t)\|_{H^{k}(\Omega)}^{2}+\int_{T_{1}}^{T_{2}}\|\nabla u(t)\|_{H^{k}(\Omega)}^{2} d t\right)^{1 / 2}<\infty .
$$

We introduce anisotropic Lebesgue and Sobolev spaces with mixed norm by setting

$$
\begin{aligned}
\|u\|_{L_{p_{2}}\left(0, T ; L_{p_{1}}(\Omega)\right)}= & \|u\|_{L_{p_{1}, p_{2}}(\Omega \times(0, T))} \\
= & \left(\int_{0}^{T}\left(\int_{\Omega}|u|^{p_{1}} d x\right)^{p_{2} / p_{1}} d t\right)^{1 / p_{2}}, \\
\|u\|_{W_{p_{1}, p_{2}}^{2,1}(\Omega \times(0, T))}= & \left\|D_{x}^{2} u\right\|_{L_{p_{1}, p_{2}}(\Omega \times(0, T))}+\left\|\partial_{t} u\right\|_{L_{p_{1}, p_{2}}(\Omega \times(0, T))} \\
& +\|u\|_{L_{p_{1}, p_{2}}(\Omega \times(0, T))}, \quad p_{1}, p_{2} \in(1, \infty) .
\end{aligned}
$$

We use the definition of Besov spaces from [BIN, Ch. 4, Sect. 18], and the theorem on direct and inverse traces between Besov and Sobolev spaces with mixed norm from $[\mathrm{B}]$.

Let us consider the elliptic problem

$$
\begin{array}{ll}
\operatorname{rot} u=h & \text { in } \Omega, \\
\operatorname{div} u=0 & \text { in } \Omega, \\
u \cdot \bar{n}=0 & \text { on } S .
\end{array}
$$

Lemma 2.1 (see [S1]). Let $h \in H^{m}(\Omega)$ for some $m \in \mathbb{N}_{0}$. Then there exists a solution $u$ to problem (2.1) such that $u \in H^{m+1}$ and

$$
\|u\|_{H^{m+1}(\Omega)} \leq c\|h\|_{H^{m}(\Omega)} .
$$

Let us consider the problem

$$
\begin{array}{ll}
\operatorname{div} \mathbb{T}(u, q)=h & \text { in } \Omega, \\
\operatorname{div} u=0 & \text { in } \Omega, \\
u=0 & \text { on } S .
\end{array}
$$

By $\mathbb{T}(u, q)$ we denote the stress tensor of the form

$$
\mathbb{T}(u, q)=\nu \mathbb{D}(u)-q I,
$$

where $\mathbb{D}(u)$ is the dilatation tensor of the form

$$
\mathbb{D}(u)=\left\{u_{i, x_{j}}+u_{j, x_{i}}\right\}_{i, j=1,2}
$$

and $I$ is the unit matrix.

Lemma 2.2 (see [S2]). Let $h \in L_{2}(\Omega)$. Then there exists a solution $(u, q)$ to problem (2.3) such that $u \in H^{2}(\Omega)$ and $\nabla q \in L_{2}(\Omega)$ and

$$
\|u\|_{H^{2}(\Omega)}+\|\nabla q\|_{L_{2}(\Omega)} \leq c\|h\|_{L_{2}(\Omega)} .
$$


We need to examine the problem

$$
\begin{array}{ll}
\operatorname{rot} \operatorname{rot} H=h & \text { in } \Omega, \\
\operatorname{div} H=0 & \text { in } \Omega, \\
H \cdot \bar{n}=0, \quad \bar{n} \times \operatorname{rot} H=0 & \text { on } S, \\
\operatorname{div} h=0 . &
\end{array}
$$

Lemma 2.3 (see [S1]). Assume that $h \in L_{2}(\Omega)$. Then there exists a solution $H$ to problem (2.5) such that $H \in H^{2}(\Omega)$ and

$$
\|H\|_{H^{2}(\Omega)} \leq c\|h\|_{L_{2}(\Omega)} .
$$

Let us consider the following initial-boundary value problem for the nonstationary Stokes system

$$
\begin{array}{ll}
v_{t}-\nu \Delta v+\nabla p=f & \text { in } \Omega^{T}, \\
\operatorname{div} v=0 & \text { in } \Omega^{T}, \\
v=0 & \text { on } S^{T}, \\
\left.v\right|_{t=0}=v_{0} & \text { in } \Omega .
\end{array}
$$

From [S3, S4, K] we have

Lemma 2.4. Assume that $f \in L_{r, \sigma}\left(\Omega^{T}\right)$ and $v_{0} \in B_{r, \sigma}^{2-2 / \sigma}(\Omega)$ for some $r, \sigma \in(1, \infty)$. Then there exists a solution $(v, p)$ to problem $(2.7)$ such that $v \in W_{r, \sigma}^{2,1}\left(\Omega^{T}\right), \nabla p \in L_{r, \sigma}\left(\Omega^{T}\right)$ and

$$
\|v\|_{W_{r, \sigma}^{2,1}\left(\Omega^{T}\right)}+\|\nabla p\|_{L_{r, \sigma}\left(\Omega^{T}\right)} \leq c\left(\|f\|_{L_{r, \sigma}\left(\Omega^{T}\right)}+\left\|v_{0}\right\|_{B_{r, \sigma}^{2-2 / \sigma}(\Omega)}\right) .
$$

Let us consider the following initial-boundary value problem for the heat equations:

$$
\begin{array}{ll}
H_{t}-\mu \Delta H=f & \text { in } \Omega^{T}, \\
H \cdot \bar{n}=0, \quad \bar{n} \times \operatorname{rot} H=0 & \text { on } S^{T}, \\
\left.H\right|_{t=0}=H_{0} & \text { in } \Omega,
\end{array}
$$

where $\bar{n}$ is the unit outward normal vector to $S$. From [DHP] we have

Lemma 2.5. Assume that $f \in L_{r, q}\left(\Omega^{T}\right)$ and $H_{0} \in B_{r, q}^{2-2 / q}(\Omega)$ for some $r, q \in(1, \infty)$. Then there exists a unique solution $H$ to problem (2.9) such that $H \in W_{r, q}^{2,1}\left(\Omega^{T}\right)$ and

$$
\|H\|_{W_{r, q}^{2,1}\left(\Omega^{T}\right)} \leq c\left(\|f\|_{L_{r, q}\left(\Omega^{T}\right)}+\left\|H_{0}\right\|_{B_{r, q}^{2-2 / q}(\Omega)}\right) .
$$

3. Axi-symmetric solutions. To obtain estimates for the axi-symmetric mhd described by problem (1.8)-(1.10) we perform all calculations exactly in the same way as for the general three-dimensional problem (1.1)-(1.4). The calculations can be done in that way because solutions to (1.8)-(1.10) 
depend on $\varphi$ through the vectors $\bar{e}_{r}, \bar{e}_{\varphi}$ only, so all the derived integral estimates are automatically restricted to $\Omega_{0}$.

First we have

Lemma 3.1. Assume that

$$
\begin{aligned}
& A_{1}^{2}=\frac{1}{\nu} \sup _{k \in \mathbb{N}_{0}} \int_{k T}^{(k+1) T}\left\|f_{s}(t)\right\|_{L_{2}}^{2} d t<\infty, \\
& A_{2}^{2}=\frac{A_{1}^{2}}{c_{s 1}\left(1-e^{-\nu_{*} c_{s 1} T}\right)}+\left\|v_{s}(0), H_{s}(0)\right\|_{L_{2}}^{2}<\infty,
\end{aligned}
$$

where $c_{s 1}$ is a constant depending on the constants from the Poincaré inequality and from (2.2), and $\nu_{*}=\min \{\nu, \mu\}$. Then

$$
\begin{aligned}
& \left\|v_{s}(k T), H_{s}(k T)\right\|_{L_{2}}^{2} \leq A_{2}^{2}, \\
& \left\|v_{s}(t), H_{s}(t)\right\|_{L_{2}}^{2}+\nu_{*} c_{s 1} \int_{k T}^{t}\left\|v_{s}\left(t^{\prime}\right), H_{s}\left(t^{\prime}\right)\right\|_{H^{1}}^{2} d t^{\prime} \leq A_{1}^{2}+A_{2}^{2} \equiv A_{3}^{2}
\end{aligned}
$$

for all $t \in(k T,(k+1) T]$ and $k \in \mathbb{N}_{0}$.

Proof. Multiplying $(1.8)_{1}$ by $v_{s},(1.8)_{3}$ by $H_{s}$, integrating over $\Omega$, using $(1.8)_{2,4}$ and the boundary conditions we obtain

$$
\frac{1}{2} \frac{d}{d t} \int_{\Omega}\left(v_{s}^{2}+H_{s}^{2}\right) d x+\int_{\Omega}\left(\nu\left|\nabla v_{s}\right|^{2}+\mu\left|\operatorname{rot} H_{s}\right|^{2}\right) d x=\int_{\Omega} f_{s} \cdot v_{s} d x .
$$

Using the Poincaré inequality for $v_{s}$, Lemma 2.1 for $H_{s}$ and applying the Hölder and Young inequalities to the r.h.s. we get

$$
\frac{d}{d t}\left(\left\|v_{s}\right\|_{L_{2}}^{2}+\left\|H_{s}\right\|_{L_{2}}^{2}\right)+\nu c_{s 1}\left\|v_{s}\right\|_{H^{1}}^{2}+\mu c_{s 1}\left\|H_{s}\right\|_{H^{1}}^{2} \leq \frac{1}{\nu c_{s 1}}\left\|f_{s}\right\|_{L_{2}}^{2} .
$$

Using $\nu_{*}=\min \{\nu, \mu\}$ we obtain

$$
\frac{d}{d t}\left[\left(\left\|v_{s}\right\|_{L_{2}}^{2}+\left\|H_{s}\right\|_{L_{2}}^{2}\right) \exp \left(\nu_{*} c_{s 1} t\right)\right] \leq \frac{1}{\nu c_{s 1}}\left\|f_{s}\right\|_{L_{2}}^{2} \exp \left(\nu_{*} c_{s 1} t\right) .
$$

Integrating with respect to time from $t=k T$ to $t \in(k T,(k+1) T]$ yields

$$
\begin{aligned}
\left\|v_{s}(t)\right\|_{L_{2}}^{2}+\left\|H_{s}(t)\right\|_{L_{2}}^{2} \leq & \frac{1}{\nu c_{s 1}} \int_{k T}^{t}\left\|f_{s}\left(t^{\prime}\right)\right\|_{L_{2}}^{2} d t^{\prime} \\
& +\exp \left(-\nu_{*} c_{s 1}(t-k T)\right)\left(\left\|v_{s}(k T)\right\|_{L_{2}}^{2}+\left\|H_{s}(k T)\right\|_{L_{2}}^{2}\right)
\end{aligned}
$$

for $t \in(k T,(k+1) T]$. Setting $t=(k+1) T$ we get

$$
\begin{aligned}
\left\|v_{s}((k+1) T), H_{s}((k+1) T)\right\|_{L_{2}}^{2} \leq & \frac{1}{\nu c_{s 1}} \int_{k T}^{(k+1) T}\left\|f_{s}(t)\right\|_{L_{2}}^{2} d t \\
& +\exp \left(-\nu_{*} c_{s 1} T\right)\left\|v_{s}(k T), H_{s}(k T)\right\|_{L_{2}}^{2} .
\end{aligned}
$$


By iteration we have

$$
\begin{aligned}
& \left\|v_{s}(k T), H_{s}(k T)\right\|_{L_{2}}^{2} \\
& \leq \frac{A_{1}^{2}}{c_{s 1}\left(1-e^{-\nu_{*} c_{s} T}\right)}+e^{-\nu_{*} c_{s 1} k T}\left\|v_{s}(0), H_{s}(0)\right\|_{L_{2}}^{2} \leq A_{2}^{2} .
\end{aligned}
$$

Integrating (3.4) with respect to time from $k T$ to $t \in(k T,(k+1) T)$ yields $(3.2)_{2}$. This concludes the proof.

LEMmA 3.2. If the assumptions of Lemma 3.1 are satisfied and $T$ is so large that

$$
-\nu_{*} c_{s 2} T / 2+A_{3}^{4} \leq 0
$$

and

$$
\begin{aligned}
& A_{4}^{2}=\exp \left(A_{3}^{4}\right) A_{1}^{2}, \\
& A_{5}^{2}=\frac{c_{s 2} A_{4}^{2}}{1-\exp \left(-\nu_{*} c_{s 2} T / 2\right)}+\left\|\nabla v_{s}(0), \operatorname{rot} H_{s}(0)\right\|_{L_{2}}^{2}<\infty,
\end{aligned}
$$

then

$$
\begin{aligned}
& \left\|v_{s}(k T), H_{s}(k T)\right\|_{H^{1}}^{2} \leq c A_{5}^{2}, \\
& \left\|v_{s}(t), H_{s}(t)\right\|_{H^{1}}^{2}+\int_{k T}^{t}\left\|v_{s}\left(t^{\prime}\right), H_{s}\left(t^{\prime}\right)\right\|_{H^{2}}^{2} d t^{\prime} \leq c c_{s 3} A_{3}^{4} A_{5}^{2}+c A_{5}^{2} \equiv c A_{6}^{2}
\end{aligned}
$$

for all $t \in(k T,(k+1) T]$ and $k \in \mathbb{N}_{0}$.

Proof. Multiplying $(1.8)_{1}$ by $\frac{1}{\nu} \operatorname{div} \mathbb{T}\left(v_{s}, q_{s}\right)$ and $(1.8)_{3}$ by $\Delta H_{s}$, integrating the results over $\Omega$ and using the boundary conditions we obtain

$$
\frac{d}{d t}\left\|\nabla v_{s}, \operatorname{rot} H_{s}\right\|_{L_{2}}^{2}+\frac{1}{\nu}\left\|\operatorname{div} \mathbb{T}\left(v_{s}, q_{s}\right)\right\|_{L_{2}}^{2}+\mu\left\|\Delta H_{s}\right\|_{L_{2}}^{2}
$$

$$
\begin{aligned}
\leq & c\left(\left\|v_{s}\right\|_{L_{2}}^{2}\left\|\nabla v_{s}\right\|_{L_{2}}^{4}+\left\|H_{s}\right\|_{L_{2}}^{2}\left\|\nabla H_{s}\right\|_{L_{2}}^{4}+\left\|v_{s}\right\|_{L_{2}}^{2}\left\|\nabla H_{s}\right\|_{L_{2}}^{4}+\left\|H_{s}\right\|_{L_{2}}^{2}\left\|\nabla v_{s}\right\|_{L_{2}}^{4}\right) \\
& +\frac{c}{\nu}\left\|f_{s}\right\|_{L_{2}}^{2} .
\end{aligned}
$$

In view of Lemmas $2.1-2.3$ we derive

$$
\begin{aligned}
\frac{d}{d t}\left\|\nabla v_{s}, \operatorname{rot} H_{s}\right\|_{L_{2}}^{2} & +c_{s 2} \nu\left\|v_{s}\right\|_{H^{2}}^{2}+\mu c_{s 2}\left\|H_{s}\right\|_{H^{2}}^{2} \\
& \leq c_{s 3}\left\|v_{s}, H_{s}\right\|_{L_{2}}^{2}\left\|\nabla v_{s}, \operatorname{rot} H_{s}\right\|_{L_{2}}^{4}+\frac{c_{s 3}}{\nu}\left\|f_{s}\right\|_{L_{2}}^{2} .
\end{aligned}
$$

Inequality (3.8) implies

$$
\begin{gathered}
\frac{d}{d t}\left[\left\|\nabla v_{s}, \operatorname{rot} H_{s}\right\|_{L_{2}}^{2} \exp \left(\nu_{*} c_{s 2} t-\sup _{t}\left\|v_{s}, H_{s}\right\|_{L_{2}}^{2} \int_{k T}^{t}\left\|\nabla v_{s}, \operatorname{rot} H_{s}\right\|_{L_{2}}^{2} d t^{\prime}\right)\right] \\
\leq \frac{c_{s 2}}{\nu}\left\|f_{s}\right\|_{L_{2}}^{2} \exp \left(\nu_{*} c_{s 2} t-\sup _{t}\left\|v_{s}, H_{s}\right\|_{L_{2}}^{2} \int_{k T}^{t}\left\|\nabla v_{s}, \operatorname{rot} H_{s}\right\|_{L_{2}}^{2} d t^{\prime}\right) .
\end{gathered}
$$


Integrating the above inequality with respect to time from $k T$ to $t \in(k T$, $(k+1) T]$ yields

$$
\begin{aligned}
& \left\|\nabla v_{s}(t), \operatorname{rot} H_{s}(t)\right\|_{L_{2}}^{2} \\
& \leq \exp \left(\sup _{t}\left\|v_{s}, H_{s}\right\|_{L_{2}}^{2} \int_{k T}^{t}\left\|\nabla v_{s}, \operatorname{rot} H_{s}\right\|_{L_{2}}^{2} d t^{\prime}\right) \frac{c_{s 2}}{\nu} \int_{k T}^{t}\left\|f_{s}\left(t^{\prime}\right)\right\|_{L_{2}}^{2} d t^{\prime} \\
& \quad+\left\|\nabla v_{s}(k T), \operatorname{rot} H_{s}(k T)\right\|_{L_{2}}^{2} \\
& \quad \cdot \exp \left(-\nu_{*} c_{s 2}(t-k T)+\sup _{t}\left\|v_{s}, H_{s}\right\|_{L_{2}}^{2} \int_{k T}^{t}\left\|\nabla v_{s}\left(t^{\prime}\right), \operatorname{rot} H_{s}\left(t^{\prime}\right)\right\|_{L_{2}}^{2} d t^{\prime}\right) .
\end{aligned}
$$

Taking into account assumptions (3.1) of Lemma 3.1 we have

$$
\begin{aligned}
\left\|\nabla v_{s}(t), \operatorname{rot} H_{s}(t)\right\|_{L_{2}}^{2} & \leq c_{s 2} \exp \left(A_{3}^{4}\right) \frac{1}{\nu} \int_{k T}^{t}\left\|f_{s}\left(t^{\prime}\right)\right\|_{L_{2}}^{2} d t^{\prime} \\
& +\left\|\nabla v_{s}(k T), \operatorname{rot} H_{s}(k T)\right\|_{L_{2}}^{2} \exp \left(-\nu_{*} c_{s 2}(t-k T)+A_{3}^{4}\right) .
\end{aligned}
$$

Setting $t=(k+1) T$, using $(3.1)_{1}$ and $(3.5)_{1}$ yields

$$
\begin{aligned}
& \left\|\nabla v_{s}((k+1) T), \operatorname{rot} H_{s}((k+1) T)\right\|_{L_{2}}^{2} \\
& \quad \leq c_{s 2} \exp \left(A_{3}^{4}\right) A_{1}^{2}+\exp \left(-\nu_{*} c_{s 2} T / 2\right)\left\|\nabla v_{s}(k T), \operatorname{rot} H_{s}(k T)\right\|_{L_{2}}^{2} .
\end{aligned}
$$

Applying the notation (3.5) 2 and iteration we have

$$
\begin{aligned}
\left\|\nabla v_{s}(k T), \operatorname{rot} H_{s}(k T)\right\|_{L_{2}}^{2} \leq & \frac{c_{s 2} A_{4}^{2}}{1-\exp \left(-\nu_{*} c_{s 2} T / 2\right)} \\
& +\exp \left(-\nu_{*} c_{s 2} T / 2\right)\left\|\nabla v_{s}(0), \operatorname{rot} H_{s}(0)\right\|_{L_{2}}^{2} \\
\leq & A_{5}^{2} .
\end{aligned}
$$

Hence $(3.6)_{1}$ is proved. Integrating (3.8) with respect to time from $k T$ to $t \in(k T,(k+1) T]$ and employing (3.9) yields (3.6) $)_{2}$. This concludes the proof.

To guarantee the assumptions of Lemma 4.2 below we have to show that $v_{s}, H_{s} \in C\left([k T,(k+1) T] ; W_{3^{+}}^{1}\left(\Omega_{0}\right)\right)$ for any $k \in \mathbb{N}_{0}$.

LEMma 3.3. Assume that there exists $\sigma>3$ such that $v_{s}(0), H_{s}(0) \in$ $B_{\sigma, 2}^{1}\left(\Omega_{0}\right)$ and $f_{s} \in L_{2}\left(k T,(k+1) T ; L_{\sigma}\left(\Omega_{0}\right)\right)$ for all $k \in \mathbb{N}_{0}$. Then $v_{s}, H_{s} \in$ $C\left(\mathbb{R}_{+} ; W_{\sigma_{0}}^{1}\left(\Omega_{0}\right)\right)$ for any $\sigma_{0}$ such that $2<\sigma_{0}<\sigma$, and

$$
\left\|v_{s}(t), H_{s}(t)\right\|_{W_{\sigma_{0}}^{1}\left(\Omega_{0}\right)} \leq c_{0},
$$

where $c_{0}$ does not depend on time.

Proof. In view of Lemma 3.2 we see that $v_{s} \cdot \nabla v_{s}, H_{s} \cdot \nabla H_{s}, v_{s} \cdot \nabla H_{s}$, $H_{s} \cdot \nabla v_{s}$ belong to $L_{2}\left(k T,(k+1) T ; L_{\sigma}\left(\Omega_{0}\right)\right)$ for some $\sigma \in(1, \infty)$. Then the assumptions of the lemma and the theory developed in [S3, S4, K] imply 
that there exists a solution to problem (1.8)-(1.10) such that $\left(v_{s}, H_{s}\right) \in$ $W_{\sigma, 2}^{2,1}\left(\Omega_{0} \times(0, T)\right)$ and

$$
\begin{aligned}
& \left\|v_{s}, H_{s}\right\|_{W_{\sigma, 2}^{2,1}\left(\Omega_{0} \times(0, T)\right)} \\
& \quad \leq c\left(A_{6}^{2}+\left\|f_{s}\right\|_{L_{2}\left(0, T ; L_{\sigma}\left(\Omega_{0}\right)\right)}+\left\|v_{s}(0), H_{s}(0)\right\|_{B_{\sigma, 2}^{1}\left(\Omega_{0}\right)}\right) .
\end{aligned}
$$

Estimate (3.11) is only valid in the interval $(0, T)$ because to prove it in any interval $(k T,(k+1) T)$ we need an estimate for $\left\|v_{s}(k T), H_{s}(k T)\right\|_{B_{\sigma, 2}^{1}\left(\Omega_{0}\right)}$ independent of $k$. Since we are not able to control this, we introduce smooth cut-off functions $\zeta_{k}=\zeta_{k}(t)$ such that $\zeta_{k}(t)=0$ for $t \in[k T, k T+\delta / 2]$ and $\zeta_{k}(t)=1$ for $t \geq k T+\delta, \delta<T$. Introducing the new quantities $\tilde{v}_{s}=v_{s} \zeta_{k}$, $\tilde{p}_{s}=p_{s} \zeta_{k}, \tilde{H}_{s}=H_{s} \zeta_{k}, f_{s}=f_{s} \zeta_{k}, \zeta_{k}=\zeta_{k, t}$ we see that $\left(\tilde{v}_{s}, \tilde{p}_{s}, \tilde{H}_{s}\right)$ is a solution to the problem

$$
\begin{aligned}
& \tilde{v}_{s t}-\nu \Delta \tilde{v}_{s}+\nabla \tilde{p}_{s}=v_{s} \dot{\zeta}_{k}-v_{s} \cdot \nabla \tilde{v}_{s}+H_{s} \cdot \nabla \tilde{H}_{s} \\
& -H_{s i} \nabla \tilde{H}_{s i}+\tilde{f}_{s} \quad \text { in } \Omega_{0} \times(k T,(k+1) T), \\
& \tilde{H}_{s t}-\mu \Delta \tilde{H}_{s}=H_{s} \dot{\zeta}_{k}-v_{s} \cdot \nabla \tilde{H}_{s}+H_{s} \cdot \nabla \tilde{v}_{s} \quad \text { in } \Omega_{0} \times(k T,(k+1) T), \\
& \operatorname{div} \tilde{v}_{s}=0, \quad \operatorname{div} \tilde{H}_{s}=0 \text {, } \\
& \left.\tilde{v}_{s}\right|_{S_{0}}=0,\left.\quad \bar{n} \cdot \tilde{H}_{s}\right|_{S_{0}}=0, \quad \bar{n} \times\left.\operatorname{rot} \tilde{H}_{s}\right|_{S_{0}}=0, \\
& \left.\tilde{v}_{s}(t)\right|_{t=k T}=0,\left.\quad \tilde{H}_{s}(t)\right|_{t=k T}=0 .
\end{aligned}
$$

From Lemmas 2.4, 2.5, 3.2 we deduce the following estimate for solutions to problem (3.12):

$$
\left\|\tilde{v}_{s}, \tilde{H}_{s}\right\|_{W_{\sigma, 2}^{2,1}(\Omega \times(k T,(k+1) T))} \leq c\left(A_{6}+A_{6}^{2}+\left\|\tilde{f}_{s}\right\|_{L_{2}\left(\Omega_{0} \times(k T,(k+1) T)\right)}\right),
$$

where the constant $c$ does not depend on $k$. Nevertheless, $c$ might depend on $T$.

To infer $(3.13)$ in the interval $[k T, k T+\delta]$ we use (3.13) for $[(k-1) T, k T]$. Then, applying [B], we derive

$$
\begin{aligned}
& \left\|v_{s}(k T)\right\|_{B_{\sigma, 2}^{1}\left(\Omega_{0}\right)} \leq c\left\|v_{s}\right\|_{W_{\sigma, 2}^{2,1}\left(\Omega_{0} \times((k-1) T, k T)\right)}, \\
& \left\|H_{s}(k T)\right\|_{B_{\sigma, 2}^{1}\left(\Omega_{0}\right)} \leq c\left\|H_{s}\right\|_{W_{\sigma, 2}^{2,1}\left(\Omega_{0} \times((k-1) T, k T)\right)} .
\end{aligned}
$$

In this way we prove (3.10) for all $t \in \mathbb{R}_{+}$with a constant $c_{0}$ independent of $k$. To prove continuity of $v_{s}, H_{s}$ with respect to time we need some $\sigma_{0}$ such that $2<\sigma_{0}<\sigma$ in order to apply imbeddings and to get the Hölder continuity. This concludes the proof.

4. Stability. To prove stability of the axi-symmetric solutions we examine problem (1.12)-(1.15). First we obtain an energy type estimate. 
Lemma 4.1. Let the assumptions of Lemmas 3.1 and 3.2 hold. Assume that

$$
\begin{aligned}
& -\frac{T}{2}+\frac{1}{c_{1} \nu_{*}} \int_{k T}^{(k+1) T}\left\|\nabla v_{s}(t), \nabla H_{s}(t)\right\|_{L_{3}}^{2} d t \leq 0, \\
& B_{1}^{2}=\sup _{k \in \mathbb{N}_{0}} \frac{1}{\nu_{*}} \int_{k T}^{(k+1) T}\|g(t)\|_{L_{2}}^{2} d t<\infty, \\
& B_{2}^{2}=\frac{c \exp \left(A_{6}^{2}\right) B_{1}^{2}}{1-\exp \left(-c_{1} \nu_{*} T / 2\right)}+\|u(0), K(0)\|_{L_{2}}^{2}<\infty,
\end{aligned}
$$

where $c_{1}$ appears in (4.6) and $A_{6}$ in (3.6). Then

$$
\begin{aligned}
& \|u(k T), K(k T)\|_{L_{2}} \leq B_{2}, \\
& \begin{array}{l}
\|u(t), K(t)\|_{L_{2}}^{2}+c_{1} \nu_{*} \int_{k T}^{t}\left\|u\left(t^{\prime}\right), K\left(t^{\prime}\right)\right\|_{H^{1}}^{2} d t^{\prime} \\
\quad \leq c \exp \left(A_{6}^{2}\right)\left(A_{6}^{2}+1\right)\left(B_{1}^{2}+B_{2}^{2}\right) \equiv B_{3}^{2}
\end{array}
\end{aligned}
$$

for all $t \in(k T,(k+1) T]$ and all $k \in \mathbb{N}_{0}$.

Proof. Multiplying (1.12) by $u$ and integrating over $\Omega$ yields

$$
\begin{aligned}
\frac{1}{2} \frac{d}{d t}\|u\|_{L_{2}}^{2}+\nu\|\nabla u\|_{L_{2}}^{2} & =-\int_{\Omega} u \cdot \nabla v_{s} \cdot u d x \\
& +\int_{\Omega}\left(K \cdot \nabla K \cdot u+K \cdot \nabla H_{s} \cdot u+H_{s} \cdot \nabla K \cdot u\right) d x+\int_{\Omega} g \cdot u d x .
\end{aligned}
$$

Multiplying (1.13) by $K$, integrating over $\Omega$ and using the boundary conditions implies

$$
\begin{aligned}
\frac{1}{2} \frac{d}{d t}\|K\|_{L_{2}}^{2}+\mu \| \operatorname{rot} & K \|_{L_{2}}^{2}=-\int_{\Omega} u \cdot \nabla H_{s} \cdot K d x \\
& +\int_{\Omega}\left(K \cdot \nabla u \cdot K+K \cdot \nabla v_{s} \cdot K+H_{s} \cdot \nabla u \cdot K\right) d x .
\end{aligned}
$$

Adding (4.3) and (4.4) gives

$$
\begin{aligned}
\frac{d}{d t}\|u, K\|_{L_{2}}^{2} & +\nu_{*}\|\nabla u, \operatorname{rot} K\|_{L_{2}}^{2}=-\int_{\Omega} u \cdot \nabla v_{s} \cdot u d x \\
& +\int_{\Omega} K \cdot \nabla v_{s} \cdot K d x+\int_{\Omega}\left[K \cdot \nabla(u \cdot K)+H_{s} \cdot \nabla(u \cdot K)\right] d x \\
& +\int_{\Omega}\left(K \cdot \nabla H_{s} \cdot u-u \cdot \nabla H_{s} \cdot K\right) d x+\int_{\Omega} g \cdot u d x .
\end{aligned}
$$


If we take into account the boundary conditions, the Poincaré inequality and Lemma 2.1, equality (4.5) yields

$$
\frac{d}{d t}\|u, K\|_{L_{2}}^{2}+c_{1} \nu_{*}\|u, K\|_{H^{1}}^{2} \leq c\left\|\nabla v_{s}, \nabla H_{s}\right\|_{L_{3}}^{2}\|u, K\|_{L_{2}}^{2}+\frac{c}{\nu_{*}}\|g\|_{L_{2}}^{2} .
$$

From (4.6) for $t \in[k T,(k+1) T]$ we have

$$
\begin{aligned}
\frac{d}{d t}\left[\|u, K\|_{L_{2}}^{2}\right. & \left.\exp \left(c_{1} \nu_{*} t-\int_{k T}^{t}\left\|\nabla v_{s}\left(t^{\prime}\right), \nabla H_{s}\left(t^{\prime}\right)\right\|_{L_{3}}^{2} d t^{\prime}\right)\right] \\
& \leq \frac{c}{\nu_{*}}\|g\|_{L_{2}}^{2} \exp \left(c_{1} \nu_{*} t-\int_{k T}^{t}\left\|\nabla v_{s}\left(t^{\prime}\right), \nabla H_{s}\left(t^{\prime}\right)\right\|_{L_{3}}^{2} d t^{\prime}\right) .
\end{aligned}
$$

Integrating (4.7) with respect to time from $k T$ to $t \in(k T,(k+1) T]$ implies (4.8) $\quad\|u(t), K(t)\|_{L_{2}}^{2}$

$$
\begin{aligned}
\leq & \exp \left(\int_{k T}^{t}\left\|\nabla v_{s}\left(t^{\prime}\right), \nabla H_{s}\left(t^{\prime}\right)\right\|_{L_{3}}^{2} d t^{\prime}\right) \frac{c}{\nu_{*}} \int_{k T}^{t}\left\|g\left(t^{\prime}\right)\right\|_{L_{2}}^{2} d t^{\prime} \\
& +\exp \left[-c_{1} \nu_{*}(t-k T)+\int_{k T}^{t}\left\|\nabla v_{s}\left(t^{\prime}\right), \nabla H_{s}\left(t^{\prime}\right)\right\|_{L_{3}}^{2} d t^{\prime}\right]\|u(k T), K(k T)\|_{L_{2}}^{2} .
\end{aligned}
$$

Setting $t=(k+1) T$ and employing $(4.1)_{1}$ yields

$$
\begin{aligned}
& \|u((k+1) T), K((k+1) T)\|_{L_{2}}^{2} \\
& \quad \leq \exp \left(\int_{k T}^{(k+1) T}\left\|\nabla v_{s}(t), \nabla H_{s}(t)\right\|_{L_{3}}^{2} d t\right) \frac{c}{\nu_{*}} \int_{k T}^{(k+1) T}\|g(t)\|_{L_{2}}^{2} d t \\
& \quad+\exp \left(-c_{1} \nu_{*} T / 2\right)\|u(k T), K(k T)\|_{L_{2}}^{2} .
\end{aligned}
$$

In view of (3.6) and $(4.1)_{2}$, inequality (4.9) simplifies to

$$
\begin{aligned}
& \|u((k+1) T), K((k+1) T)\|_{L_{2}}^{2} \\
& \quad \leq c \exp \left(A_{6}^{2}\right) B_{1}^{2}+\exp \left(-c_{1} \nu_{*} T / 2\right)\|u(k T), K(k T)\|_{L_{2}}^{2} .
\end{aligned}
$$

Hence, iteration yields

$$
\begin{aligned}
& \|u(k T), K(k T)\|_{L_{2}}^{2} \\
& \quad \leq \frac{c \exp \left(A_{6}^{2}\right) B_{1}^{2}}{1-\exp \left(-c_{1} \nu_{*} T / 2\right)}+\exp \left(-c_{1} \nu_{*} k T / 2\right)\|u(0), K(0)\|_{L_{2}}^{2} \leq B_{2}^{2} .
\end{aligned}
$$

This implies $(4.2)_{1}$. From (4.8) we have

$$
\|u(t), K(t)\|_{L_{2}}^{2} \leq c \exp \left(A_{6}^{2}\right)\left[B_{1}^{2}+B_{2}^{2}\right]
$$


for all $t \in(k T,(k+1) T]$ and $k \in \mathbb{N}_{0}$. Integrating (4.6) with respect to time from $k T$ to $t \in(k T,(k+1) T]$ and using (4.11) gives

$$
\begin{aligned}
\|u(t), K(t)\|_{L_{2}}^{2}+c_{1} \nu_{*} \int_{k T}^{t}\left\|u\left(t^{\prime}\right), K\left(t^{\prime}\right)\right\|_{H^{1}}^{2} d t^{\prime} \\
\leq c \sup _{t \in[k T,(k+1) T]}\|u(t), K(t)\|_{L_{2}(\Omega)}^{2} \int_{k T}^{t}\left\|\nabla v_{s}\left(t^{\prime}\right), \nabla H_{s}\left(t^{\prime}\right)\right\|_{L_{3}}^{2} d t^{\prime} \\
\quad+\frac{c}{\nu_{*}} \int_{k T}^{t}\left\|g\left(t^{\prime}\right)\right\|_{L_{2}}^{2} d t^{\prime}+\|u(k T), K(k T)\|_{L_{2}}^{2} \\
\leq c\left(A_{6}^{2}+1\right) \exp \left(A_{6}^{2}\right)\left[B_{1}^{2}+B_{2}^{2}\right] .
\end{aligned}
$$

Employing $(3.6)_{2}$ in (4.12) yields $(4.2)_{2}$. This concludes the proof.

To show stability we need

Lemma 4.2. Let the assumptions of Lemma 4.1 hold. Consider $\gamma \leq \gamma_{*}$, where $\gamma_{*}$ is so small that

$$
\gamma_{*}^{4} \leq \nu_{*}^{2} / 2
$$

Assume that

$$
\begin{aligned}
& \|\nabla u(0), \operatorname{rot} K(0)\|_{L_{2}}^{2} \leq \gamma, \\
& \frac{c_{3}}{\nu_{*}}\left\|v_{s}(t), H_{s}(t)\right\|_{W_{3}^{1}}^{4} B_{3}^{2}+\|g(t)\|_{L_{2}}^{2} \leq \nu_{*} c_{2} \frac{\gamma}{4},
\end{aligned}
$$

where the constants $c_{2}, c_{3}$ appear in (4.21) and (4.22), respectively, and $B_{3}$ is introduced in Lemma 4.1. Then

$$
\|\nabla u(t), \operatorname{rot} K(t)\|_{L_{2}}^{2} \leq \gamma \quad \text { for all } t \in \mathbb{R}_{+} .
$$

Proof. We multiply (1.12) by $\operatorname{div} \mathbb{T}(u, d)$, integrate the result over $\Omega$, use the boundary conditions and Lemma 2.2. This gives

$$
\begin{aligned}
& \nu \frac{d}{d t}\|\nabla u\|_{L_{2}}^{2}+c_{2}\left(\nu^{2}\|u\|_{H^{2}}^{2}+\|\nabla d\|_{L_{2}}^{2}\right) \\
& \leq c \int_{\Omega}\left(|u \cdot \nabla u|^{2}+\left|u \cdot \nabla v_{s}\right|^{2}+\left|v_{s} \cdot \nabla u\right|^{2}\right) d x \\
& \quad+c \int_{\Omega}\left(|K \cdot \nabla K|^{2}+\left|K \cdot \nabla H_{s}\right|^{2}+\left|H_{s} \cdot K\right|^{2}\right) d x+c\|g\|_{L_{2}}^{2} .
\end{aligned}
$$

Similarly, multiplying (1.13) by $\Delta K$, integrating over $\Omega$, using the boundary conditions and Lemma 2.3, we obtain

$$
\begin{aligned}
\frac{d}{d t}\|\operatorname{rot} K\|_{L_{2}}^{2} & +\mu\|K\|_{H^{2}}^{2} \leq c \int_{\Omega}\left(|u \cdot \nabla K|^{2}+\left|u \cdot \nabla H_{s}\right|^{2}\right. \\
& \left.+\left|v_{s} \cdot \nabla K\right|^{2}+|K \cdot \nabla u|^{2}+\left|K \cdot \nabla v_{s}\right|^{2}+\left|H_{s} \cdot \nabla u\right|^{2}\right) d x .
\end{aligned}
$$


First we estimate the terms from the r.h.s. of (4.15). Using the interpolation inequality (see [BIN, Ch. 3, Sect. 15])

$$
\|\nabla u\|_{L_{3}} \leq c\|u\|_{H^{2}}^{1 / 2}\|\nabla u\|_{L_{2}}^{1 / 2} \leq \varepsilon\|u\|_{H^{2}}+\frac{c}{\varepsilon}\|\nabla u\|_{L_{2}}
$$

we obtain

$$
\begin{aligned}
\int_{\Omega}|u \cdot \nabla u|^{2} d x & \leq\|u\|_{L_{6}}^{2}\|\nabla u\|_{L_{3}}^{2} \leq c\|u\|_{H^{1}}^{2}\|u\|_{H^{2}}\|u\|_{H^{1}} \\
& \leq \varepsilon_{1}\|u\|_{H^{2}}^{2}+\frac{c}{\varepsilon_{1}}\|u\|_{H^{1}}^{6} \leq \varepsilon_{1}\|u\|_{H^{2}}^{2}+\frac{c}{\varepsilon_{1}}\|u\|_{L_{2}}^{6} .
\end{aligned}
$$

In view of the interpolation inequality

$$
\|\nabla u\|_{L_{2}} \leq \varepsilon^{1 / 2}\left\|\nabla^{2} u\right\|_{L_{2}}+c \varepsilon^{-1 / 2}\|u\|_{L_{2}}
$$

and the Poincaré inequality it follows that

$$
\begin{aligned}
\int_{\Omega}\left|u \cdot \nabla v_{s}\right|^{2} d x & \leq\|u\|_{L_{6}}^{2}\left\|\nabla v_{s}\right\|_{L_{3}}^{2} \leq c\|u\|_{H^{1}}^{2}\left\|\nabla v_{s}\right\|_{L_{3}}^{2} \\
& \leq c\|\nabla u\|_{L_{2}}^{2}\left\|\nabla v_{s}\right\|_{L_{3}}^{2} \leq\left(\varepsilon\left\|\nabla^{2} u\right\|_{L_{2}}^{2}+\frac{c}{\varepsilon}\|u\|_{L_{2}}^{2}\right)\left\|\nabla v_{s}\right\|_{L_{3}}^{2} \\
& \leq \varepsilon_{2}\left\|\nabla^{2} u\right\|_{L_{2}}^{2}+\frac{c}{\varepsilon_{2}}\left\|\nabla v_{s}\right\|_{L_{3}}^{4}\|u\|_{L_{2}}^{2} .
\end{aligned}
$$

Similarly, (4.18) implies

$$
\begin{aligned}
\int_{\Omega}\left|v_{s} \cdot \nabla u\right|^{2} d x & \leq\left\|v_{s}\right\|_{L_{\infty}}^{2}\|\nabla u\|_{L_{2}}^{2} \leq\left(\varepsilon\left\|\nabla^{2} u\right\|_{L_{2}}^{2}+\frac{c}{\varepsilon}\|u\|_{L_{2}}^{2}\right)\left\|v_{s}\right\|_{L_{\infty}}^{2} \\
& \leq \varepsilon_{3}\left\|\nabla^{2} u\right\|_{L_{2}}^{2}+\frac{c}{\varepsilon_{3}}\left\|v_{s}\right\|_{L_{\infty}}^{4}\|u\|_{L_{2}}^{2} .
\end{aligned}
$$

Repeating the above estimates we derive

$$
\begin{aligned}
& \int_{\Omega}|K \cdot \nabla K|^{2} d x \leq \varepsilon_{4}\|K\|_{H^{2}}^{2}+\frac{c}{\varepsilon_{4}}\|K\|_{H^{1}}^{6}, \\
& \int_{\Omega}\left|K \cdot \nabla H_{s}\right|^{2} d x \leq \varepsilon_{5}\left\|\nabla^{2} K\right\|_{L_{2}}^{2}+\frac{c}{\varepsilon_{5}}\left\|\nabla H_{s}\right\|_{L_{3}}^{4}\|K\|_{L_{2}}^{2}, \\
& \int_{\Omega}\left|H_{s} \cdot \nabla K\right|^{2} d x \leq \varepsilon_{6}\left\|\nabla^{2} K\right\|_{L_{2}}^{2}+\frac{c}{\varepsilon_{6}}\left\|H_{s}\right\|_{L_{\infty}}^{4}\|K\|_{L_{2}}^{2} .
\end{aligned}
$$

In view of the above estimates and for sufficiently small $\varepsilon_{1}-\varepsilon_{6}$ we obtain from (4.13) the inequality

$$
\begin{aligned}
& \frac{d}{d t}\|\nabla u\|_{L_{2}}^{2}+c_{2} \nu\|u\|_{H^{2}}^{2} \\
\leq & \bar{\varepsilon}_{1}\|u, K\|_{H^{2}}^{2}+\frac{c}{\bar{\varepsilon}_{1}}\|u, K\|_{H^{1}}^{6}+\frac{c}{\bar{\varepsilon}_{1}}\left(\left\|\nabla v_{s}\right\|_{L_{3}}^{4}\|u\|_{L_{2}}^{2}+\left\|\nabla K_{s}\right\|_{L_{3}}^{4}\|K\|_{L_{2}}^{2}\right) \\
& +\frac{c}{\bar{\varepsilon}_{1}}\left(\left\|v_{s}\right\|_{L_{\infty}}^{4}\|u\|_{L_{2}}^{2}+\left\|H_{s}\right\|_{L_{\infty}}^{4}\|K\|_{L_{2}}^{2}\right)+\frac{c}{\nu}\|g\|_{L_{2}}^{2} .
\end{aligned}
$$


Similarly, we estimate the integrals from the r.h.s. of (4.16) by

$$
\begin{aligned}
& \int_{\Omega}|u \cdot \nabla K|^{2} d x \leq\|u\|_{L_{6}}^{2}\|\nabla K\|_{L_{3}}^{2} \leq \varepsilon_{7}\|K\|_{H^{2}}^{2}+\frac{c}{\varepsilon_{7}}\|u\|_{H^{1}}^{4}\|\nabla K\|_{L_{2}}^{2}, \\
& \int_{\Omega}\left|u \cdot \nabla H_{s}\right|^{2} d x \leq \varepsilon_{8}\left\|\nabla^{2} u\right\|_{L_{2}}^{2}+\frac{c}{\varepsilon_{8}}\left\|\nabla H_{s}\right\|_{L_{3}}^{4}\|u\|_{L_{2}}^{2}, \\
& \int_{\Omega}\left|v_{s} \cdot \nabla K\right|^{2} d x \leq \varepsilon_{9}\left\|\nabla^{2} K\right\|_{L_{2}}^{2}+\frac{c}{\varepsilon_{9}}\left\|v_{s}\right\|_{L_{\infty}}^{4}\|K\|_{L_{2}}^{2}, \\
& \int_{\Omega}|K \cdot \nabla u|^{2} d x \leq\|K\|_{L_{6}}^{2}\|\nabla u\|_{L_{3}}^{2} \leq \varepsilon_{10}\|u\|_{H^{2}}^{2}+\frac{c}{\varepsilon_{10}}\|K\|_{H^{1}}^{4}\|\nabla u\|_{L_{2}}^{2}, \\
& \int_{\Omega}\left|K \cdot \nabla v_{s}\right|^{2} d x \leq\|K\|_{L_{6}}^{2}\left\|\nabla v_{s}\right\|_{L_{3}}^{2} \leq \varepsilon_{11}\left\|\nabla^{2} K\right\|_{L_{2}}^{2}+\frac{c}{\varepsilon_{11}}\left\|\nabla v_{s}\right\|_{L_{3}}^{4}\|K\|_{L_{2}}^{2}, \\
& \int_{\Omega}\left|H_{s} \cdot \nabla u\right|^{2} d x \leq \varepsilon_{12}\left\|\nabla^{2} u\right\|_{L_{2}}^{2}+\frac{c}{\varepsilon_{12}}\left\|H_{s}\right\|_{L_{\infty}}^{4}\|u\|_{L_{2}}^{2} .
\end{aligned}
$$

Employing the above estimates in (4.16) yields

$$
\begin{aligned}
& \frac{d}{d t}\|\operatorname{rot} K\|_{L_{2}}^{2}+\mu\|K\|_{H^{2}}^{2} \leq \bar{\varepsilon}_{2}\|u, K\|_{H^{2}}^{2} \\
& \quad+\frac{c}{\varepsilon_{2}}\left(\|u, K\|_{H^{1}}^{6}+\left\|\nabla H_{s}\right\|_{L_{3}}^{2}\|u\|_{L_{2}}^{2}+\left\|\nabla v_{s}\right\|_{L_{3}}^{2}\|K\|_{L_{2}}^{2}\right. \\
& \left.\quad+\left\|v_{s}\right\|_{L_{\infty}}^{4}\|K\|_{L_{2}}^{2}+\left\|H_{s}\right\|_{L_{\infty}}^{4}\|u\|_{L_{2}}^{2}\right) .
\end{aligned}
$$

Adding (4.19) and (4.20) with sufficiently small $\bar{\varepsilon}_{1}$ and $\bar{\varepsilon}_{2}$ and employing Lemma 2.1 gives

$$
\begin{aligned}
& \frac{d}{d t}\|\nabla u, \operatorname{rot} K\|_{L_{2}}^{2}+c_{2} \nu_{*}\|u, K\|_{H^{2}}^{2} \leq \frac{c}{\nu_{*}}\|u, K\|_{H^{1}}^{6} \\
& \quad+\frac{c}{\nu_{*}}\left(\left\|\nabla v_{s}, \nabla H_{s}\right\|_{L_{3}}^{4}+\left\|v_{s}, H_{s}\right\|_{L_{\infty}}^{4}\right)\|u, K\|_{L_{2}}^{2}+\frac{c}{\nu}\|g\|_{L_{2}}^{2} .
\end{aligned}
$$

In view of the Poincaré inequality and Lemma 2.1 we have

$$
\begin{aligned}
& \frac{d}{d t}\|\nabla u, \operatorname{rot} K\|_{L_{2}}^{2}+c_{2} \nu_{*}\|u, K\|_{H^{2}}^{2} \leq \frac{c_{3}}{\nu_{*}}\|\nabla u, \operatorname{rot} K\|_{L_{2}}^{6} \\
& +\frac{c_{3}}{\nu_{*}}\left(\left\|\nabla v_{s}, \nabla H_{s}\right\|_{L_{3}}^{4}+\left\|v_{s}, H_{s}\right\|_{L_{\infty}}^{4}\right)\|u, K\|_{L_{2}}^{2}+\frac{c_{3}}{\nu}\|g\|_{L_{2}}^{2} .
\end{aligned}
$$

Using $(4.2)_{2}$ and introducing the notation

$$
\begin{aligned}
& X(t)=\|\nabla u(t), \operatorname{rot} K(t)\|_{L_{2}}, \\
& Y(t)=\|u(t), K(t)\|_{H^{2}}, \\
& G^{2}(t)=\frac{c_{3}}{\nu_{*}}\left(\left\|\nabla v_{s}(t), \nabla H_{s}(t)\right\|_{L_{3}}^{4} B_{3}^{2}+\left\|v_{s}(t), H_{s}(t)\right\|_{L_{\infty}}^{4} B_{3}^{2}+\|g\|_{L_{2}}^{2}\right),
\end{aligned}
$$


we express (4.22) in the short form

$$
\frac{d}{d t} X^{2} \leq-\nu_{*} c_{2} Y^{2}+\frac{c_{3}}{\nu_{*}} X^{6}+G^{2}(t) .
$$

Since $X \leq Y$, we have

$$
\frac{d}{d t} X^{2} \leq-X^{2}\left(\nu_{*} c_{2}-\frac{c_{3}}{\nu_{*}} X^{4}\right)+G^{2}(t) .
$$

Let $\gamma \in\left(0, \gamma_{*}\right]$, where $\gamma_{*}$ is so small that

$$
\nu_{*} c_{2}-\frac{c_{2}}{\nu_{*}} \gamma_{*}^{4} \geq \nu_{*} \frac{c_{2}}{2} .
$$

By the assumptions of the lemma,

$$
X^{2}(0) \leq \gamma, \quad G^{2}(t) \leq \nu_{*} c_{2} \frac{\gamma}{4} \quad \text { for all } t \in \mathbb{R}_{+} .
$$

Suppose that

$$
t_{*}=\inf \left\{t \in \mathbb{R}_{+}: X^{2}(t)>\gamma\right\}>0 .
$$

By (4.24), for $t \in\left(0, t_{*}\right]$ inequality (4.23) takes the form

$$
\frac{d}{d t} X^{2} \leq-\frac{\nu_{*} c_{2}}{2} X^{2}+G^{2}(t) .
$$

By definition of $t_{*}$ we have

$$
X^{2}\left(t_{*}\right)=\gamma \quad \text { and } \quad X^{2}(t)>\gamma \quad \text { for } t>t_{*} .
$$

Then (4.25) yields

$$
\left.\frac{d}{d t} X^{2}(t)\right|_{t=t_{*}} \leq \nu_{*} c_{2}\left(-\frac{\gamma}{2}+\frac{\gamma}{4}\right)<0,
$$

contradicting (4.26). Therefore

$$
X^{2}(t)<\gamma \quad \text { for } t \in \mathbb{R}_{+} .
$$

This concludes the proof.

Acknowledgments. The research leading to these results has received funding from the People Programme (Marie Curie Actions) of the European Union's Seventh Framework Programme FP7/2007-2013/ under REA grant agreement no. 319012 and from the Funds for International Co-operation under Polish Ministry of Science and Higher Education grant agreement no. $2853 / 7 . P R / 2013 / 2$.

\section{References}

[BIN] O. V. Besov, V. P. Il'in and S. M. Nikolskii, Integral representations of functions and imbedding theorems, Nauka, Moscow, 1975 (in Russian).

[B] Ya. S. Bugrov, Function spaces with mixed norm, Math. USSR-Izv. 5 (1971), 11451167. 
[CW] C. Cao and J. Wu, Global regularity for the 2DMHD equations with mixed dissipation and magnetic diffusion, Adv. Math. 226 (2011), 1802-1822.

[CMZ] Q. Chen, C. Miao and Z. Zhang, On the regularity criterion of weak solution for the $3 D$ viscous magneto-hydrodynamics equations, Comm. Math. Phys. 284 (2008), 919-930.

[C] G. H. A. Cole, Fluid Dynamics, Methuen, London, 1962.

[DHP] R. Denk, M. Hieber and J. Prüss, Optimal $L_{p}-L_{q}$ estimates for parabolic boundary value problems with inhomogeneous data, Math. Z. 257 (2007), 193-224.

[DL] G. Duvaut and J.-L. Lions, Inéquations en thermoélasticité et magnétohydrodynamique, Arch. Ration. Mech. Anal. 46 (1971), 241-279.

[HX1] C. He and X. Xin, Partial regularity of suitable weak solutions to the incompressible magnetohydrodynamic equations, J. Funct. Anal. 227 (2005), 113-152.

[HX2] C. He and X. Xin, On the regularity of weak solutions to magnetohydrodynamic equations, J. Differential Equations 213 (2005), 235-254.

[K] N. V. Krylov, On the Calderón-Zygmund theorem with applications to parabolic equations, Algebra i Analiz 13 (2001), no. 4, 1-25 (in Russian).

[La1] O. A. Ladyzhenskaya, On unique solvability of three-dimensional Cauchy problem for the Navier-Stokes equations under axial symmetry, Zap. Nauchn. Sem. LOMI 7 (1968), 155-177 (in Russian).

[La2] O. A. Ladyzhenskaya, Solutions 'in the large' of the nonstationary boundary value problem for the Navier-Stokes system with two space variables, Comm. Pure Appl. Math. 12 (1959), 427-433.

[LLP] L. D. Landau, E. M. Lifshitz and L. P. Pitayevskii, Electrodynamics of Continuous Media, 2nd ed., Landau and Lifshitz Course of Theoretical Physics, vol. 8.

[L] Z. Lei, On axially symmetric incompressible magnetohydrodynamics in three dimensions, J. Differential Equations 259 (2015), 3202-3215.

[LXZ] F. Lin, L. Xu and P. Zhang, Global small solutions of 2-D incompressible magnetohydrodynamics system, J. Differential Equations 259 (2015), 5440-5485.

[LZ] F. Lin and P. Zhang, Global small solutions to an MHD-type system: the threedimensional case, Comm. Pure Appl. Math. 67 (2014), 531-580.

[NSZ] B. Nowakowski, G. Ströhmer and W. M. Zajączkowski, Large time existence of special strong solutions to mhd equations in cylindrical domains, submitted.

[NZ] B. Nowakowski and W. M. Zajączkowski, On global regular solutions to magnetohydrodynamics in axi-symmetric domains, Z. Angew. Math. Phys. 67 (2016), no. 6, art. $142,22 \mathrm{pp}$.

[ST] M. Sermange and R. Temam, Some mathematical questions related to the MHD equations, Comm. Pure Appl. Math. 36 (1983), 635-664.

[S1] V. A. Solonnikov, Overdetermined elliptic boundary value problems, Zap. Nauchn. Sem. LOMI 21 (1971), 112-158 (in Russian).

[S2] V. A. Solonnikov, On general boundary value problems for the Douglis-Nirenberg elliptic systems, Izv. Akad. Nauk SSSR Ser. Mat. 28 (1964), 665-706; Trudy MIAN SSSR 92 (1966), 233-297 (in Russian).

[S3] V. A. Solonnikov, Estimates of solutions of the Stokes equations in Sobolev spaces with a mixed norm, Zap. Nauchn. Sem. POMI 288 (2002), 204-231.

[S4] V. A. Solonnikov, On estimates of solutions of the nonstationary Stokes problem in anisotropic Sobolev spaces and on estimates of the resolvent of the Stokes problem, Uspekhi Mat. Nauk 58 (2003), no. 2, 123-156 (in Russian).

[XZ] L. Xu and P. Zhang, Global small solutions to three-dimensional incompressible magnetohydrodynamical system, SIAM J. Math. Anal. 47 (2015), 26-65. 
[ZZ1] E. Zadrzyńska and W. M. Zajączkowski, Stability of two-dimensional NavierStokes motions in the periodic case, J. Math. Anal. Appl. 423 (2015), 956-974.

[ZZ2] E. Zadrzyńska and W. M. Zajączkowski, Stability of two-dimensional heat-conducting incompressible motion in a cylinder, Nonlinear Anal. 125 (2015), 113-127.

[Z1] W. M. Zajączkowski, Stability of nonswirl axisymmetric solutions to the NavierStokes equations, in: Mathematical Analysis of Viscous Incompressible Fluid, RIMS Kôkyûroku No. 2009, 2016, 84-104.

[Z2] W. M. Zajączkowski, Stability of two-dimensional solutions to the Navier-Stokes equations in cylindrical domains under Navier boundary conditions, J. Math. Anal. Appl. 444 (2016), 275-297.

[Z3] W. M. Zajączkowski, Global special regular solutions to the Navier-Stokes equations in a cylindrical domain without the axis of symmetry, Topol. Methods Nonlinear Anal. 24 (2004), 69-105.

[Z] T. Zhang, An elementary proof of the global existence and uniqueness theorem to 2-D incompressible non-resistive MHD system, arXiv:1404.5681 (2014).

Alexander Mikhaylov

Steklov Institute of Mathematics

Fontanka 7

St. Petersburg, Russia

Wojciech M. Zajączkowski

Institute of Mathematics

Polish Academy of Sciences

Śniadeckich 8

00-656 Warszawa, Poland

and

Institute of Mathematics and Cryptology

Cybernetics Faculty

Military University of Technology

Kaliskiego 2

00-908 Warszawa, Poland

E-mail:wz@impan.pl
Joanna Rencławowicz Institute of Mathematics Polish Academy of Sciences Śniadeckich 8 00-656 Warszawa, Poland E-mail: jr@impan.pl 
